> Le cerveau, via la régulation de la sensation de faim, contrôle la prise alimentaire et l'homéostasie du glucose. II possède en particulier deux structures spécifiques, l'hypothalamus et le tronc cérébral, sensibles à des informations provenant des organes périphériques ou de l'intestin (par l'intermédiaire d'hormones ou de substances nutritives circulantes), qui le renseignent sur l'état nutritionnel de l'organisme. Récemment, un nouvel axe nerveux intestin-cerveau a été identifié. Certains nutriments contrôlent la fonction homéostatique hypothalamique par cet axe. Nous décrivons dans cet article les connexions nerveuses entre l'intestin et le cerveau, et leur rôle dans l'homéostasie énergétique. <

Depuis que Claude Bernard a montré que des lésions du plancher du Iv ventricule induisent très rapidement un diabète chez le lapin, on connaît la capacité de l'encéphale à réguler la glycémie. Cela a été très étudié chez des modèles animaux. Par exemple, de nombreuses études ont montré que le cerveau possède des neurones détecteurs de glucose, qui réagissent aux variations de glycémie [1]. Des mécanismes de signalisation centrale permettent, notamment, d'activer une machinerie cellulaire semblable à celle des cellules $\beta$-pancréatiques, mettant en jeu notamment des canaux $K_{\text {ATP }}$ [1]. Le cerveau semble donc être un élément clé dans la régulation de la glycémie. II est ainsi particulièrement sensible à l'état énergétique de l'organisme, notamment à travers l'apport en glucose.

Ainsi, I'AMP-activated protein kinase (AMPK), une enzyme essentielle pour l'homéostasie énergétique, qui régule des processus énergétiques périphériques (oxydation des substrats glucidiques et lipidiques en particulier), est présente dans l'hypothalamus. Elle est activée notamment en cas de carence en glucose [2]. Au-delà de la détection du glucose et de la régulation de la glycémie, le cerveau est impliqué de façon cruciale dans le contrôle de la prise alimentaire et

\section{Contrôle de la glycémie par l'axe nerveux intestin-cerveau}

Filipe De Vadder, Gilles Mithieux

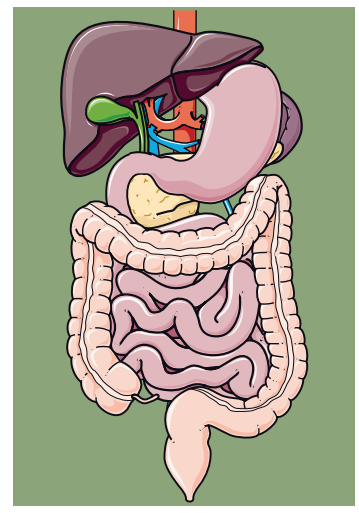

Inserm U855, 7-11 rue Paradin, 69372 Lyon Cedex 08, France; Université de Lyon, 69008 Lyon, France ; Université Lyon 1, 69622 Villeurbanne, France. gilles.mithieux@inserm.fr

de la balance énergétique. Les résultats de la dernière décennie ont d'ailleurs conduit à l'idée selon laquelle ce sont les mêmes processus centraux qui régulent l'apport de glucose dans le sang depuis l'extérieur (via la prise alimentaire, modulée par la sensation de faim) et l'intérieur du corps (via la production endogène de glucose [PEG], modulée par la balance sympathique). La découverte de la leptine, une hormone sécrétée par le tissu adipeux, qui diminue la sensation de faim en se liant à des récepteurs présents dans l'hypothalamus, en est une parfaite illustration. La leptine est en effet capable également de réguler la production endogène de glucose par des mécanismes dépendants et indépendants de l'hypothalamus [3, 4]. L'hypothalamus et le tronc cérébral, où se connectent la moelle épinière et le système nerveux périphérique (nerfs vagues), occupent une place centrale dans ces régulations [5]. Cette revue portera, d'une part, sur les mécanismes centraux impliqués dans la régulation de la glycémie et, d'autre part, sur les signaux provenant de l'intestin qui interviennent dans cette modulation.

\section{Intégration des signaux de régulation par l'hypothalamus et le tronc cérébral}

L'hypothalamus et le tronc cérébral sont les régions historiquement les plus étudiées pour le contrôle de la prise alimentaire et du métabolisme énergétique en général. Ces deux régions contiennent des organes circumventriculaires (éminence médiane et area postrema [AP]) qui possèdent des capillaires fenestrés et une perméabilité plus grande aux métabolites circulants que les autres structures cérébrales, qui, elles, sont isolées de la circulation par la barrière hémato-encéphalique [6, 31]. Le tronc cérébral, quant à lui, est le siège de l'intégration des informations nerveuses issues du système nerveux périphérique, notamment 
les afférences gustatives (via le nerf glosso-pharyngien ou nerf crânien $\mathrm{IX}$ ) et les afférences vagales (Figure 1 ). II constitue un centre intégrateur des informations, ensuite relayées vers le reste du cerveau et, plus particulièrement, l'hypothalamus. Étudiés avant tout pour leur rôle dans les sensations de faim et de satiété, I'hypothalamus et le tronc cérébral contrôlent aussi le métabolisme glucidique.

\section{Les noyaux de l'hypothalamus}

L'identification des neurotransmetteurs dans ces noyaux a confirmé le rôle central de l'hypothalamus, mais a surtout révélé une grande diversité neuronale. L'hypothalamus des mammifères est constitué de plus de 40 noyaux histologiquement distincts, chacun divisé en sousnoyaux. La zone médiane est essentiellement composée de grands noyaux (noyau dorso-médian [DMN], noyau ventro-médian [VMN]), qui reçoivent des informations sensorielles et sont fortement interconnectés avec le reste de l'hypothalamus. Cette zone est impliquée dans l'organisation des comportements adaptatifs. La zone latérale (LHA) possède un système de communication intra- et extra-hypothalamique; elle peut être définie comme l'interface entre les régions plus médianes et les régions cortico-limbiques d'une part, et entre les systèmes moteurs somatique et autonome d'autre part. Le noyau paraventriculaire (PVN) de l'hypothalamus représente un microcosme à l'intérieur de l'hypothalamus, dans le sens où plusieurs sous-noyaux sont connectés aux trois systèmes effecteurs: le système endocrine (groupes magnocellulaires), le système autonome et le système comportemental (groupes parvocellulaires) [5].

De nombreuses études, depuis une vingtaine d'années, ont cherché à identifier les populations neuronales hypothalamiques qui contiennent des neurotransmetteurs et des récepteurs spécifiques d'importance cruciale dans le comportement alimentaire et la régulation de la glycémie. Parmi les noyaux hypothalamiques, le noyau arqué (ARC) occupe un rôle intégrateur majeur, avec des connexions à la zone latérale, au tronc cérébral et au système cortico-limbique, entre autres [7] (Figure 1). Plusieurs populations neuronales ont été identifiées dans l'hypothalamus, sur la base de leur contenu en neurotransmetteurs. Dans le noyau arqué, deux populations neuronales principales répondent rapidement aux informations nutritionnelles. L'une exprime le gène codant pour la pro-opiomélanocortine (POMC) et le transcrit régulé par la cocaïne et les amphétamines (CART); elle induit un effet anorexigène [8]. La forme active de POMC est le résultat de son clivage en plusieurs neuropeptides, parmi lesquels les hormones mélanotropes $\alpha$ et $\beta$ ( $\alpha$ - et $\beta$-MSH), qui exercent leur effet anorexigène par leur liaison aux récepteurs MC4R (melanocortin 4 receptor) et MC3R [9]. Contrairement aux neurones POMC/CART, I'autre population de neurones du noyau arqué coexprime le neuropeptide $Y$ (NPY) et l'agouti-related peptide (AgRP) ; elle induit un effet orexigène [10]. Ces neurones s'opposent à l'action des neurones POMC/CART, notamment par l'effet antagoniste de l'AgRP sur les récepteurs MC3/4R [10]. Les neurones du noyau arqué expriment de multiples récepteurs d'hormones. La leptine [4], mais aussi l'insuline et les métabolites tels que le glucose ou les acides gras [1], ont directement accès au noyau arqué au niveau de l'éminence médiane [31].
Régulation du comportement alimentaire par le tronc cérébral

La deuxième composante essentielle de ce système homéostatique contrôlant la prise alimentaire et la glycémie est le tronc cérébral [11]. Le tronc cérébral contient de nombreuses voies sensitives et motrices en provenance et à destination des viscères. L'intégration des signaux renseignant le centre nerveux sur l'état énergétique de l'organisme a également lieu dans cette région de l'encéphale.

Le complexe dorsal vagal est la partie caudale du tronc cérébral ; celle-ci est composée du noyau du tractus solitaire (NTS), de l'area postrema (AP) et du noyau dorsal moteur du nerf vague (DMNX). Depuis le début des années 1990, des études ont montré que le tronc cérébral est capable d'organiser certains aspects du comportement alimentaire en l'absence d'information hypothalamique [12]. Hormis le noyau arqué, le noyau du tractus solitaire est une des seules régions du cerveau à posséder une population de neurones à POMC. Le noyau du tractus solitaire et le noyau dorsal moteur du nerf vague ont les concentrations les plus élevées de MC4R dans l'encéphale [11]. Le complexe dorsal vague exprime également fortement le récepteur à la leptine [11]. Une autre similitude avec l'hypothalamus est la possibilité que l'area postrema (ainsi que certaines parties très vascularisées du noyau du tractus solitaire) puisse détecter des hormones circulantes et d'autres facteurs. Ainsi, l'information transmise par les hormones gastro-intestinales accède directement au noyau du tractus solitaire par l'intermédiaire de récepteurs dans l'area postrema et des nombreuses projections de celle-ci au noyau du tractus solitaire (Figure 1). De plus, comme l'hypothalamus, le noyau du tractus solitaire possède des neurones sensibles au glucose [11].

C'est après avoir intégré tous ces signaux que I'hypothalamus et le tronc cérébral sont en mesure de réguler la glycémie, finement et rapidement, en mobilisant les systèmes nerveux efférents sympathique et parasympathique portés par le nerf vague et les nerfs sympathiques. Un effet majeur prend place au niveau $\mathrm{du}$ foie et de la production endogène de glucose. Le tonus sympathique augmente la glycémie en activant la glycogénolyse hépatique, tandis que le tonus parasympathique stoppe la production hépatique de glucose, en particulier en stimulant le stockage de glycogène [13]. Une dérégulation de ces mécanismes de contrôle est donc susceptible d'altérer de façon déterminante l'homéostasie glucidique et d'installer les conditions du pré-diabète. 


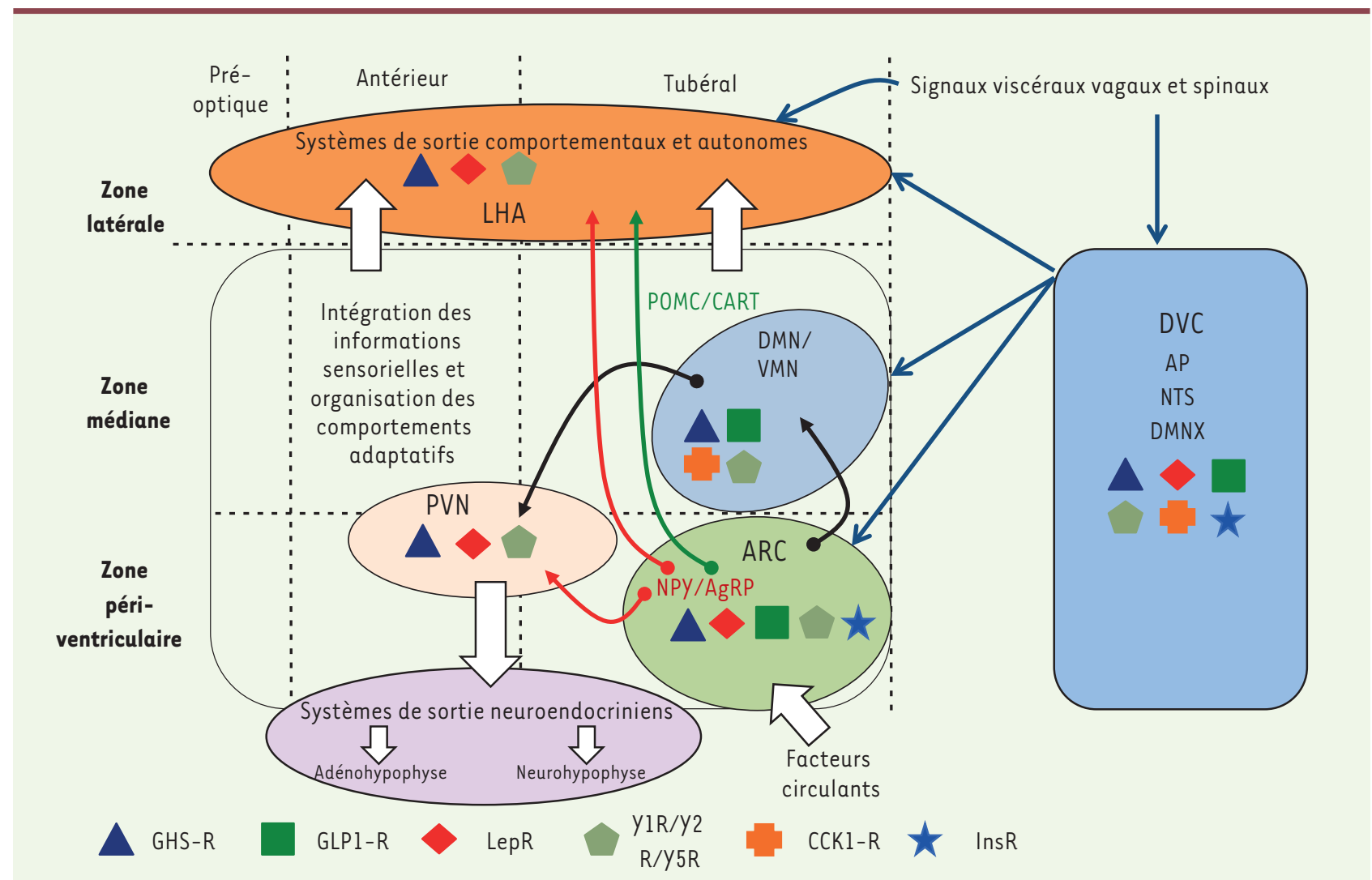

Figure 1. Organisation anatomique et fonctionnelle de l'hypothalamus et du complexe dorsal vagal (DVC) (d'après [14]). Carte fonctionnelle de I'hypothalamus et du DVC avec les éléments de détection humorale et nerveuse. AP : area postrema ; ARC : noyau arqué de l'hypothalamus ; CART : transcrit régulé par la cocaïne et les amphétamines; DMNX : noyau moteur dorsal du nerf vague; DMN/VMN : noyaux dorso-médian/ventro-médian de l'hypothalamus; LHA : hypothalamus latéral ; NTS : noyau du tractus solitaire ; POMCT : pro-opiomélanocortine ; PVN : noyau paraventriculaire de I'hypothalamus. Les sites d'expression des récepteurs de la ghréline (GHS-R), du GLP-l (glucagon-like peptide l) (GLPI-R), de la leptine (LepR), de la CCK1 (cholecystokinin 1) (CCK1-R), de l'insuline (InsR) et des NPy (neuropeptide Y)/Pyy (peptide YY) (YlR/Y2R/Y5R) sont indiqués (d'après [14]).

\section{L'axe nerveux intestin-cerveau dans le contrôle de l'homéostasie énergétique}

\section{L'innervation des viscères}

L'innervation des viscères par le système nerveux autonome se fait par les nerfs vagues et splanchniques (Figure 2). Les nerfs vagues (nerfs crâniens $X$ ) émergent principalement du tronc cérébral et transmettent des informations efférentes, mais aussi des influx sensitifs provenant des viscères thoraciques et abdominaux, des barorécepteurs de la crosse de l'aorte, des chémorécepteurs de la crosse de l'aorte, des glomus carotidiens (chémorécepteurs pour la respiration), et des calicules gustatifs de la partie postérieure de la langue. Les deux nerfs vagues (ventral et dorsal) fournissent des neurofibres au cou et aux plexus nerveux, qui desservent pratiquement tous les organes des cavités thoracique et abdominale [14]. Les nerfs vagues innervent le foie, la vésicule biliaire, l'estomac, l'intestin grêle, les reins, le pancréas et la partie proximale du gros intestin (le rectum étant innervé par les nerfs splanchniques pelviens). Ils transmettent essentiellement les signaux parasympathiques.
L'innervation sympathique de l'abdomen est assurée par des neurofibres post-ganglionnaires provenant de T5 (cinquième nerf thoracique) à L2 (deuxième nerf lombaire), qui empruntent certains nerfs splanchniques et font synapse, principalement dans les ganglions cœliaque et mésentérique supérieur [23]. Les neurofibres issues de ces ganglions rejoignent habituellement leurs organes cibles en compagnie des artères qui les desservent. Elles innervent ainsi l'estomac, les intestins (sauf la moitié distale du gros intestin), le foie, la rate et les reins (Figure 2). Comme les nerfs vagues, les nerfs splanchniques transmettent aussi des signaux sensitifs à destination du centre nerveux.

La détection intestinale et/ou portale des nutriments La zone hépato-portale possède une triple innervation périphérique assurée par les deux branches du nerf vague et le nerf splanchnique [14]. La veine porte, qui rassemble les veines mésentériques irriguant l'intestin sur toute sa longueur, est en position idéale pour 


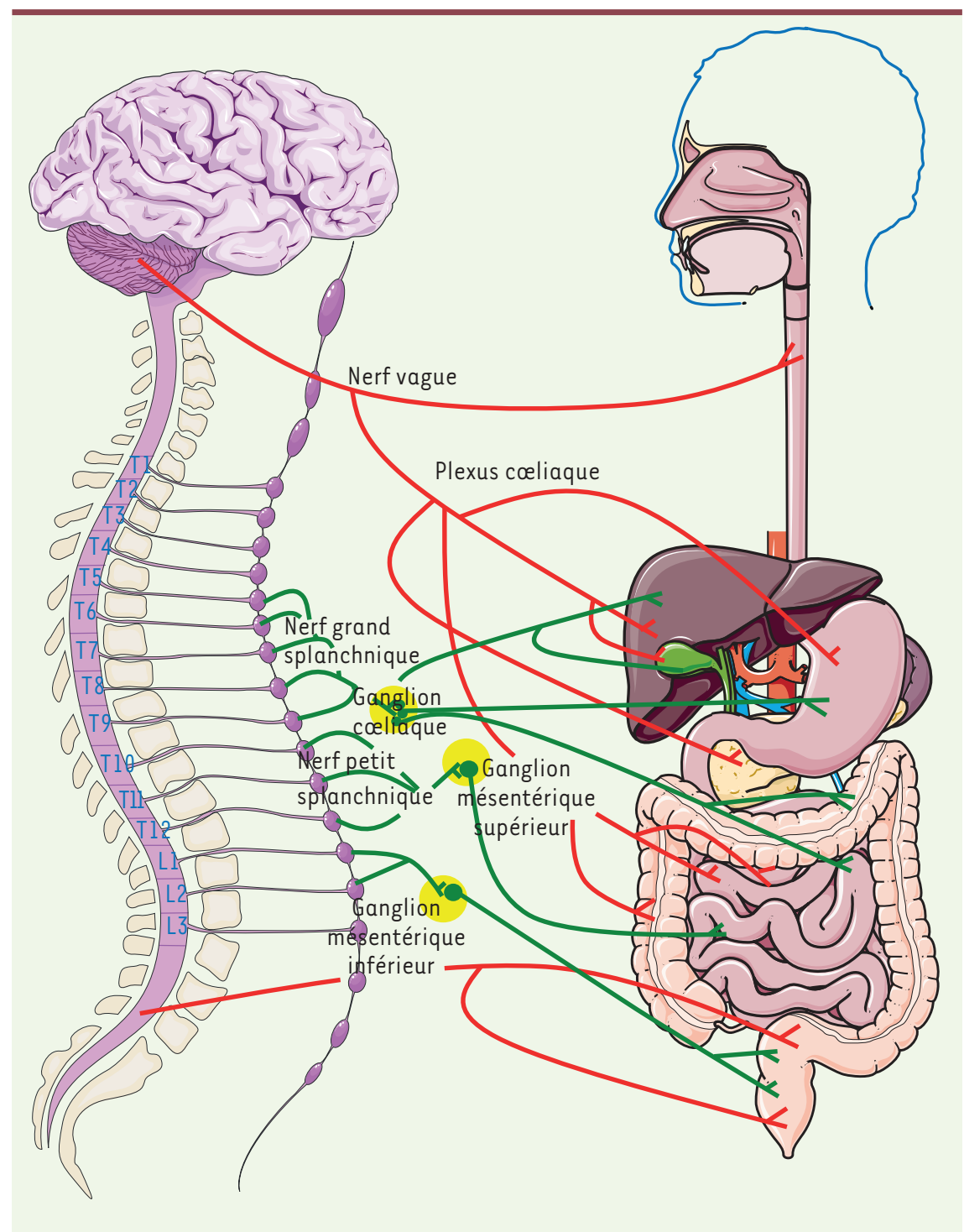

Figure 2. Innervation du tractus gastrointestinal par le système nerveux autonome. Le système nerveux sympathique est indiqué en vert, le parasympathique en rouge (illustration réalisée avec l'aide de Servier medical art).

ces mécanismes sont, en particulier, à l'origine des effets rapides sur la régulation de la glycémie observés après un by-pass gastrique chez les rongeurs $[18,19]$.

\section{Rôle clé du glucose portal}

Le flux d'apparition du glucose dans la veine porte lors de la digestion d'un repas riche en glucides (environ $50 \%$ des apports caloriques) est élevé. II représenterait une à deux fois l'équivalent de la production endogène de glucose totale [17]. La perfusion d'un tel flux de glucose induit des réponses physiologiques et comportementales complexes, telles qu'une diminution de la prise alimentaire, une modification de la préférence alimentaire, et une modification de l'activité électrique des afférences hépato-portales vagales et spinales, associées à un changement de l'activité des neurones hypothalamiques impliqués dans le contrôle de la faim [17]. Cependant, la perfusion à des flux nettement inférieurs (environ un sixième à un tiers de

détecter les informations nutritionnelles issues des repas, avant que les nutriments ne soient métabolisés par le foie. En accord avec ce rôle important de détection, les parois de la veine porte sont traversées par un réseau très dense d'astrocytes et de neurones depuis la périphérie de la veine jusqu'au lumen sanguin [15].

Bien que les nutriments soient capables d'induire, dans la période suivant leur absorption, des réponses influençant la prise alimentaire et le métabolisme après le passage dans le foie, seule la région hépatoportale est exposée à l'ensemble des signaux issus de l'alimentation ; tous les autres organes reçoivent ces informations après une utilisation au moins partielle des nutriments dans le foie, le cœur et les poumons. Un apport de lipides dans le lumen intestinal ou de glucose dans la veine porte, pendant (dans le cas des lipides) ou peu après (dans le cas du glucose) la digestion d'un repas, active les neurones localisés dans la paroi de la veine porte pour le glucose, ou dans le système nerveux gastro-intestinal au sens large pour les lipides [16]. Cela permet au cerveau de moduler aussi bien l'appétit que le flux de glucose produit par le foie (pour revue récente, [17]). On pense que la production endogène de glucose) est suffisante pour déclencher, à la fois, une diminution de la prise alimentaire et une activation des régions hypothalamiques contrôlant l'homéostasie énergétique [20]. Bien que les deux flux de glucose portal puissent représenter ce qui a lieu en période post-prandiale, il a été clairement établi que l'arrivée, même massive, de glucose dans la veine porte ne détermine pas la fin d'un repas en cours, mais conditionne plutôt la taille du repas suivant [21]. Cela suggère fortement une relation entre détection $\mathrm{du}$ glucose portal et satiété. En accord avec cette hypothèse, la région portale est un lieu crucial de détection d'une hypoglycémie d'installation lente [22], qui pourrait mimer la transition entre un l'état nourri et l'état qui est observé dans la période suivant l'absorption.

Il est intéressant de mentionner qu'un grand nombre d'arguments suggèrent un rôle clé du récepteur de glucose/cotransporteur de sodium SGLT3 (sodium-glucose 
linked transporter 3) - et non du transporteur de glucose Glut2, comme on le pense généralement - dans la détection de l'apparition du glucose portal à de faibles flux physiologiques. En effet, la détection de glucose portal est inhibée par la phlorizine (un inhibiteur spécifique des protéines de la famille des SGLT), alors que la perfusion de 3-0-méthylglucose (un analogue du glucose non phosphorylable), qui est capable de se lier à SGLT3, active le système de détection du glucose portal [15]. Autre observation remarquable: alors que la région portale est innervée par les deux voies vagale et spinale [14], la détection portale de glucose n'est pas altérée par une ablation chirurgicale du nerf vague [15]. Le signal porté par le glucose portal pourrait être relayé par les fibres afférentes des deux voies vagale et spinale, alors qu'on pense généralement que c'est la voie vagale qui transmet les signaux afférents au cerveau [14].

La détection portale de glucose revêt une importance particulière, car ce processus est mis en jeu par deux types de nutriments et est requis pour que ces nutriments exercent leurs effets. En effet, ce système a permis d'expliquer les effets bénéfiques associés aux régimes riches en protéines [20,23] ou en fibres solubles [24]. II a ainsi été récemment démontré que l'effet de satiété, bien connu lors des régimes enrichis en protéines, est indirectement transmis au cerveau via l'activation de la néoglucogenèse intestinale et d'un signal glucose portal. Ce signal prend place pendant la période suivant l'absorption; ceci a permis de comprendre le fait que le glucose exerce des effets de satiété et non de rassasiement [20]. De façon intéressante, l'activation de ce signal se traduit aussi par des effets bénéfiques sur la sensibilité à l'insuline de la production endogène de glucose [25]. Sur le plan mécanistique, la détection portale préalable des peptides par les récepteurs $\mu$-opioïdes de la paroi de la veine porte et l'arc réflexe nerveux est impliquée dans l'induction de la néoglucogenèse intestinale, mettant en exergue à nouveau le rôle clé du système nerveux périphérique et du cerveau [23]. De même, l'induction de la néoglucogenèse intestinale a permis d'expliquer les effets anti-obésité et anti-diabète des fibres alimentaires, qui sont fermentées en métabolites intermédiaires puis en acides gras à courte chaîne (propionate et butyrate) par le microbiote intestinal [30] ; ces effets sont connus depuis très longtemps, mais restent inexpliqués au plan des mécanismes. Ici encore, un premier circuit nerveux veine porte-cerveau, engagé par le récepteur des acides gras FFAR3 (free fatty acid receptor 3) (GPR41) présent dans le système nerveux périportal [32], permet d'expliquer l'effet du propionate en termes d'induction de l'expression des gènes de la néoglucogenèse intestinale [24]. Le propionate sert également de précurseur pour le glucose produit par la néoglucogenèse intestinale. Le butyrate, quant à lui, induit l'expression des gènes contrôlant la néoglucogenèse intestinale par un mécanisme direct lié à son effet de substrat énergétique pour les cellules intestinales, et non par une communication intestin-cerveau. Dans un deuxième temps, le glucose produit exerce ses effets bénéfiques sur la dépense énergétique de repos et la sensibilité à l'insuline via le signal glucose au niveau portal [24]. Ainsi, l'effet anti-obésité et anti-diabète des fibres alimentaires met en lumière la complexité des interactions entre le métabolisme intestinal des nutriments et le système nerveux péri-intestinal et central, et l'importance de ces interactions sur la régulation de l'homéostasie énergétique en général et de la glycémie en particulier. Enfin, il faut souligner que dans le foie, s'ajoutant aux régulations passant par l'expression génique, des régulations biochimiques de l'activité de la glucose- 6 phosphatase prennent place, en particulier sous I'influence des nutriments [26-29]. Si ces dernières prenaient place aussi au niveau de l'intestin, cela pourrait rendre encore plus complexe et diversifiée la façon dont la néoglucogenèse intestinale et le signal glucose portal pourraient influencer le contrôle glycémique via leur action sur l'hypothalamus. Cette hypothèse mérite certainement d'être considérée. $\diamond$

\section{SUMMARY}

Glucose homeostasis and gut-brain connection

Since the XIXth century, the brain has been known for its role in regulating food intake (via the control of hunger sensation) and glucose homeostasis. Further interest has come from the discovery of gut hormones, which established a clear link between the gut and the brain in regulating glucose and energy homeostasis. The brain has two particular structures, the hypothalamus and the brainstem, which are sensitive to information coming either from peripheral organs or from the gut (via circulating hormones or nutrients) about the nutritional status of the organism. However, the efforts for a better understanding of these mechanisms have allowed to unveil a new gut-brain neural axis as a key regulator of the metabolic status of the organism. Certain nutrients control the hypothalamic homeostatic function via this axis. In this review, we describe how the gut is connected to the brain via different neural pathways, and how the interplay between these two organs drives the energy balance. $\diamond$

\section{REMERCIEMENTS}

Le travail relatif à la référence [24] a été soutenu par une bourse de l'institut Bonduelle.

\section{LIENS D'INTÉRÊT}

Les auteurs déclarent n'avoir aucun lien d'intérêt concernant les données publiées dans cet article.

\section{RÉFÉRENCES}

1. Thorens B, Larsen PJ. Gut-derived signaling molecules and vagal afferents in the control of glucose and energy homeostasis. Curr Opin Clin Nutr Metab Care $2004 ; 7: 471-8$.

2. Andreelli F. L'AMP-activated protein kinase hypothalamique, régulateur essentiel du poids et de la prise alimentaire. Med Sci (Paris) $2005 ; 21$ : 131-2.

3. Andreelli F, Foretz M, Knauf C, et al. Liver adenosine monophosphateactivated kinase-alpha2 catalytic subunit is a key target for the control of hepatic glucose production by adiponectin and leptin but not insulin. Endocrinology 2006 ; 147 : 2432-41.

4. Gutiérrez-Juárez R, Obici S, Rossetti L. Melanocortin-independent effects of leptin on hepatic glucose fluxes. J Biol Chem $2004 ; 279$ : 49704-15. 


\section{RÉFÉRENCES}

5. Berthoud HR. Multiple neural systems controlling food intake and body weight. Neurosci Biobehav Rev $2002 ; 26$ : 393-428.

6. Duvernoy HM, Risold Py. The circumventricular organs: an atlas of comparative anatomy and vascularization. Brain Res Rev 2007 ; 56 : 119-47.

7. Guan JL, Saotome T, Wang $P P$, et al. Orexinergic innervation of POMC-containing neurons in the rat arcuate nucleus. Neuroreport $2001 ; 12: 547-51$.

8. Boston BA, Blaydon KM, Varnerin J, et al. Independent and additive effects of central POMC and leptin pathways on murine obesity. Science 1997 ; 278 : 1641-4.

9. Adan RA, Cone RD, Burbach JP, et al. Differential effects of melanocortin peptides on neural melanocortin receptors. Mol Pharmacol $1994 ; 46: 1182-90$.

10. Ollmann MM, Wilson BD, Yang YK, et al. Antagonism of central melanocortin receptors in vitro and in vivo by agouti-related protein. Science $1997 ; 278: 135-8$.

11. Blouet C. Le rôle du noyau du tractus solitaire dans la détection et l'intégration de multiples signaux métaboliques. Med Sci (Paris) $2013 ; 29: 449-52$.

12. Williams DL, Kaplan JM, Grill HJ. The role of the dorsal vagal complex and the vagus nerve in feeding effects of melanocortin-3/4 receptor stimulation. Endocrinology $2000 ; 141$ : 1332-7.

13. Uyama N, Geerts A, Reynaert H. Neural connections between the hypothalamus and the liver. Anat Rec A Discov Mol Cell Evol Biol $2004 ; 280: 808-20$.

14. Berthoud H-R. Anatomy and function of sensory hepatic nerves. Anat Rec A Discov Mol Cell Evol Biol $2004 ; 280: 827-35$.

15. Delaere F, Duchampt A, Mounien L, et al. The role of sodium-coupled glucose co-transporter 3 in the satiety effect of portal glucose sensing. Mol Metab $2012 ; 2: 47-53$.

16. Delaere F, Akaoka H, Vadder F De, et al. Portal glucose influences the sensory, cortical and reward systems in rats. Eur J Neurosci $2013 ; 38: 3476-86$.

17. Delaere F, Magnan C, Mithieux G. Hypothalamic integration of portal glucose signals and control of food intake and insulin sensitivity. Diabetes Metab $2010 ; 36: 257-62$

18. Breen DM, Rasmussen BA, Kokorovic A, et al. Jejunal nutrient sensing is required for duodenaljejunal bypass surgery to rapidly lower glucose concentrations in uncontrolled diabetes. Nat Med $2012 ; 18: 950-5$

19. Troy S, Soty M, Ribeiro L, et al. Intestinal gluconeogenesis is a key factor for early metabolic changes after gastric bypass but not after gastric lap-band in mice. Cell Metab $2008 ; 8$ : 201-11.

20. Mithieux G, Misery P, Magnan C, et al. Portal sensing of intestinal gluconeogenesis is a mechanistic link in the diminution of food intake induced by diet protein. Cell Metab $2005 ; 2: 321-9$.

21. Baird JP, Grill HJ, Kaplan JM. Intake suppression after hepatic portal glucose infusion: all-or-none effect and its temporal threshold. Am J Physiol 1997 ; 272 : R1454-60.
22. Saberi M, Bohland M, Donovan CM. The locus for hypoglycemic detection shifts with the rate of fall in glycemia: the role of portal-superior mesenteric vein glucose sensing. Diabetes $2008 ; 57: 1380-6$

23. Duraffourd C, De Vadder F, Goncalves D, et al. Mu-opioid receptors and dietary protein stimulate a gut-brain neural circuitry limiting food intake. Cell $2012 ; 150: 377-88$.

24. De Vadder F, Kovatcheva-Datchary P, Goncalves D, et al. Microbiotagenerated metabolites promote metabolic benefits via gut-brain neural circuits. Cell $2014 ; 156: 84-96$.

25. Pillot B, Soty M, Gautier-Stein A, et al. Protein feeding promotes redistribution of endogenous glucose production to the kidney and potentiates its suppression by insulin. Endocrinology 2009 ; 150 : 616-24

26. Minassian C, Mithieux G. Differential time course of liver and kidney glucose-6 phosphatase activity during fasting in rats. Comp Biochem Physiol B Biochem Mol Biol 1994 ; 109 : 99-104.

27. Daniele N, Rajas F, Payrastre B, et al. Phosphatidylinositol 3-kinase translocates onto liver endoplasmic reticulum and may account for the inhibition of glucose-6-phosphatase during refeeding. J Biol Chem 1999 ; 274: 3597-601.

28. Mithieux G, Vega FV, Riou JP. The liver glucose-6-phosphatase of intact microsomes is inhibited and displays sigmoid kinetics in the presence of alpha-ketoglutarate-magnesium and oxaloacetate-magnesium chelates. J Biol Chem $1990 ; 265: 20364-8$.

29. Mithieux G, Zitoun C. Mechanisms by which fatty-acyl-CoA esters inhibit or activate glucose-6-phosphatase in intact and detergent-treated rat liver microsomes. Eur J Biochem $1996 ; 235: 799-803$

30. El Kaoutari A, Armougom F, Raoult D, Henrissat B. Le microbiote intestinal et la digestion des polysaccharides. Med Sci (Paris) $2014 ; 30: 259-65$.

31. Balland $\varepsilon$, Prévot $V$. Les tanycytes hypothalamiques, porte d'entrée de la leptine dans le cerveau. Med Sci (Paris) 2014 ; 30 : 624-7.

32. Mancini $A$, Poitout $V$. Les récepteurs membranaires des acides gras de la cellule $\beta$. Med Sci (Paris) 2013 ; 29 : 715-21.

\section{TIRÉS À PART}

G. Mithieux

\section{First European Meeting on Bone Marrow Adiposity (1 ${ }^{\text {re }}$ réunion européenne sur l'adiposité médullaire)}

28-29 août 2015

Faculté dentaire, Lille, France

Adiposité médullaire et son environnement

Caractérisation et imagerie, physiopathologie et perspectives thérapeutiques

Site : http://bma2015.sciencesconf.org/

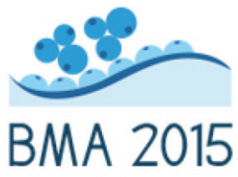

First European Meeting on Bone Marrow Adiposity

From marrow adipocytes to clinical issues

Lille, France / August 28-29, 2015

\section{Contacts :}

Pierre Hardouin, M.D., Ph.D.

Physiopathologie des Maladies Osseuses

Inflammatoires, Lille 2, ULCO, IFR 114

E-mail : pierre.hardouin@univ-littoral.fr
Pierre J. Marie, Ph.D., D.Sc.

UMR-1132 Inserm et Université Paris Diderot, Sorbonne Paris Cité

E-mail : pierre.marie@inserm.fr 